\title{
The Concordance and Heritability of Type 2 Diabetes in 34,166 Twin Pairs From International Twin Registers: The Discordant Twin (DISCOTWIN) Consortium
}

\author{
Gonneke Willemsen, ${ }^{1}$ Kirsten J. Ward, ${ }^{2}$ Christopher G. Bell, ${ }^{2}$ Kaare Christensen, ${ }^{3}$ Jocelyn Bowden, ${ }^{4}$ \\ Christine Dalgård, ${ }^{3}$ Jennifer R. Harris, ${ }^{5}$ Jaakko Kaprio, ${ }^{6,7,8}$ Robert Lyle, ${ }^{9}$ Patrik K.E. Magnusson, ${ }^{10}$ \\ Karen A. Mather, ${ }^{4}$ Juan R. Ordoňana, ${ }^{11,12}$ Francisco Perez-Riquelme, ${ }^{12}$ Nancy L. Pedersen, ${ }^{10}$ \\ Kirsi H. Pietiläinen, ${ }^{6,13,14}$ Perminder S. Sachdev, ${ }^{4}$ Dorret I. Boomsma, ${ }^{1}$ and Tim Spector ${ }^{2}$ \\ ${ }^{1}$ Department of Biological Psychology, VU University Amsterdam, Amsterdam, the Netherlands \\ ${ }^{2}$ Department of Twin Research \& Genetic Epidemiology, King's College London, London, UK \\ ${ }^{3}$ Department of Public health, Environmental Medicine, University of Southern Denmark, Odense, Denmark \\ ${ }^{4}$ Centre for Healthy Brain Ageing, School of Psychiatry, University of New South Wales, Sydney, NSW, Australia \\ ${ }^{5}$ Norwegian Twin Registry, Division of Epidemiology, The Norwegian Institute of Public Health, Oslo, Norway \\ ${ }^{6}$ Finnish Twin Cohort Study, Department of Public Health, University of Helsinki, Helsinki, Finland \\ ${ }^{7}$ Institute for Molecular Medicine FIMM, University of Helsinki, Helsinki, Finland \\ ${ }^{8}$ National Institute for Health and Welfare, Helsinki, Finland \\ ${ }^{9}$ Department of Medical Genetics, Oslo University Hospital, Oslo, Norway \\ ${ }^{10}$ Department of Medical Epidemiology and Biostatistics, Karolinska Institutet, Stockholm, Sweden \\ ${ }^{11}$ Murcia Twin Registry, Department of Human Anatomy and Psychobiology, University of Murcia, Murcia, Spain \\ ${ }^{12}$ Murcia Institute for Biomedical Research, IMIB-Arrixaca, Murcia, Spain \\ ${ }^{13}$ Obesity Research Unit, Research Programs Unit, Diabetes and Obesity, University of Helsinki, Helsinki, Finland \\ ${ }^{14}$ Endocrinology, Abdominal Center, Helsinki University Hospital and University of Helsinki, Helsinki, Finland
}

Twin pairs discordant for disease may help elucidate the epigenetic mechanisms and causal environmental factors in disease development and progression. To obtain the numbers of pairs, especially monozygotic (MZ) twin pairs, necessary for in-depth studies while also allowing for replication, twin studies worldwide need to pool their resources. The Discordant Twin (DISCOTWIN) consortium was established for this goal. Here, we describe the DISCOTWIN Consortium and present an analysis of type 2 diabetes (T2D) data in nearly 35,000 twin pairs. Seven twin cohorts from Europe (Denmark, Finland, Norway, the Netherlands, Spain, Sweden, and the United Kingdom) and one from Australia investigated the rate of discordance for T2D in same-sex twin pairs aged 45 years and older. Data were available for 34,166 same-sex twin pairs, of which 13,970 were MZ, with T2D diagnosis based on self-reported diagnosis and medication use, fasting glucose and insulin measures, or medical records. The prevalence of T2D ranged from $2.6 \%$ to $12.3 \%$ across the cohorts depending on age, body mass index (BMI), and national diabetes prevalence. T2D discordance rate was lower for $\mathrm{MZ}$ (5.1\%, range 2.9-11.2\%) than for same-sex dizygotic (DZ) (8.0\%, range 4.9-13.5\%) pairs. Across DISCOTWIN, 720 discordant MZ pairs were identified. Except for the oldest of the Danish cohorts (mean age 79), heritability estimates based on contingency tables were moderate to high (0.47-0.77). From a meta-analysis of all data, the heritability was estimated at $72 \%$ (95\% confidence interval 61-78\%). This study demonstrated high T2D prevalence and high heritability for T2D liability across twin cohorts. Therefore, the number of discordant MZ pairs for T2D is limited. By combining national resources, the DISCOTWIN Consortium maximizes the number of discordant MZ pairs needed for in-depth genotyping, multi-omics, and phenotyping studies, which may provide unique insights into the pathways linking genes to the development of many diseases.

- Keywords: discordant twins, international comparison, type 2 diabetes, heterogeneity, prevalence

RECEIVED 4 August 2015; ACCEPTED 3 September 2015.

ADDRESS FOR CORRESPONDENCE: Gonneke Willemsen, Department of Biological Psychology, VU University Amsterdam, van der Boechorststraat 1, 1081BT Amsterdam, the Netherlands. E-mail: a.h.m.willemsen@vu.nl 
Twin studies have been instrumental in determining the extent to which genes and environment influence individual differences for a wide array of diseases and diseaserelated traits, as well as for many normal behaviors and traits. Twin registers have been established to create a more long-term and stable framework for research (Hur \& Craig, 2013), aimed at elucidating genetic and environmental influences on health and development. In recognition of the need for collaboration, there has been increased networking of twin study groups, as illustrated by the International Network of Twin Registries (Buchwald et al., 2014). Twin data are also useful in collaborations that involve non-twin samples. For example, twin data have been included in many consortia and genome-wide association (GWA) studies, often providing the largest numbers within the GWA consortia.

Recent advances in molecular and omic methodologies have greatly increased the utility of the twin design for uncovering mechanisms and pathways that underlie disease development (van Dongen et al., 2012). Comparing MZ twins who are discordant for a particular trait or disease provides a unique case-control study, where the cases and controls are matched perfectly for age, sex, and genetic background, as well as for the influence of in utero and early environmental factors known to affect health and developmental trajectories. Not only may these comparisons provide insight into the influence of, for example, differential exposures, behavioral choices and life events, but when DNA is available for the twins, the contribution of epigenetic factors may also be explored. Despite the full identity of the genomic sequence in twins from $\mathrm{MZ}$ pairs, MZ twins often differ in concordance for complexly determined health and behavioral outcomes, such as diabetes, cancer, cardiovascular disease, or mental health. Epigenetic factors are one possible mechanism that could explain these intra-pair differences. The addition of data from DZ DISCOTWIN pairs and other family members helps to elucidate the causal directions within those pathways (de Moor et al., 2008; Kujala et al., 2002).

Scarcity of discordant pairs is the main hindrance to more prominent use of the co-twin control design. For any given disease or trait under study, the number of discordant MZ twin pairs is often limited as a function of heritability and with increasing prevalence of the disorder (Smith, 1970). As in genome-wide association studies (GWAS) consortia, collaboration is the best way to increase numbers, such that fully powered epigenetic studies are possible. Many of twin registers already have strong connections, in particular the European and Australian twin registers, which as part of the fifth European Framework Program (Quality of Life and Management of the Living Resources, 1998-2002; European Commission, 2004) worked together within the GenomEUtwin consortium (Peltonen \& GenomEUtwin, 2003). This collaboration led to the publication of comparative twin analyses for various traits, in- cluding body composition (Kettunen et al., 2009; Perola et al., 2007; Schousboe et al., 2003; Silventoinen et al., 2003), exercise participation (Stubbe et al., 2006; Vink et al., 2011), migraine (Mulder et al., 2003), subarachnoid hemorrhage (Korja et al., 2010), and cholesterol (Surakka et al., 2012). With this past collaborative success in mind, a new twin consortium has now been created specially focusing on discordant twins: the DISCOTWIN consortium. In addition to extensive longitudinal data on health and lifestyle, information on biomarkers, and genotypic information is also available within these cohorts, enabling the study of the effect of unique environmental influences as well as epigenetic studies.

As a demonstration project, we examined twin discordance for T2D. T2D is a complex disease, caused by insufficient pancreatic beta-cell action and/or progressive insulin resistance, and related to increasing adiposity, inactivity, and age (McCarthy, 2010). It leads to a significantly reduced life expectancy and, due to its chronic multi-systemic comorbidity, is a massive drain on healthcare resources (Zimmet et al., 2001). In 2014, the World Health Organization (WHO, 2015) estimated the global prevalence of diabetes to be $9 \%$ among adults aged 18 years or over, with $\sim 90 \%$ of these due to T2D (http://www.who.int/ mediacentre/factsheets/fs312/en/). The dramatic increase in prevalence of $\mathrm{T} 2 \mathrm{D}$ in the last 50 years is strongly driven by the rise of the modern 'obesogenic' environment, with the associated rapid upsurge in obesity due to the combined effects of reduced physical activity and increased access to high calorific foods (Friedman, 2009). These serious escalating T2D prevalence rates, while first appearing in developed Western nations, are now rapidly emerging within the developing world as they increasingly adopt modern urban lifestyles (Gluckman et al., 2011). Heritability plays a role in T2D, with estimates from twin and family studies ranging from $25 \%$ up to $69 \%$, depending upon age and glycemic cut-offs (Almgren et al., 2011; Poulsen et al., 1999). GWAS have now confirmed 92 common single nucleotide polymorphism (SNP) T2D-risk associations, including genes potentially implicated in both insulin secretion and insulin resistance, as well as unknown mechanisms of pathogenicity (Bonnefond \& Froguel, 2015).

For adequate prevention and treatment protocols, more insight into the pathways from genes to T2D development is needed. The use of twin-pairs discordant for T2D presents a unique way to disentangle environmental, genetic, and epigenetic causes of the disease. Concordance rates for T2D have been estimated in numerous studies over the years, and these have varied for multiple possible reasons, including gene pool differences, regional variation, the influence of a changing prevalence over time, age at comparison, as well as different diagnostic criteria. In an early study to distinguish between T1D and T2D, as opposed to previous childhoodand adult-onset diabetes categories, Barnett et al. (1981) observed 48 of 53 (90.6\%) MZ twin pairs to be concor- 
dant for T2D in the United Kingdom. In 1987, an American study estimated an MZ concordance rate of $58 \%$ for the National Heart, Lung, and Blood Institute Twin Study, with a population-expected prevalence at that time of $10 \%$ (Newman et al., 1987), while a Japanese study (Committee on Diabetic Twins, 1988) showed that for $83 \%$ of MZ twins with T2D, their co-twin could also be diagnosed, while this was only $40 \%$ in the DZ twins. Subsequently, in a Finnish study including 505 twins, Kaprio et al. (1992) found a probandwise $34 \%$ and pairwise $20 \% \mathrm{MZ}$ concordance, compared to $16 \%$ and $9 \%$ respectively in DZ twins. In the United Kingdom, MZ concordance rates for twin pairs who were initially ascertained as disease-discordant, were $17 \%, 33 \%$, $57 \%$, and $76 \%$ after $1,5,10$, and 15 years of follow-up, respectively (Medici et al., 1999), clearly demonstrating the effect of age and necessity for significant follow-up. The concordance rate was $96 \%$ when twins were classified for any glucose abnormality by 15 years. More recent data gathered in 2010 from a Finnish population-based study encompassing 1,332 twins ( $\mathrm{MZ}$ and $\mathrm{DZ}$ ) who developed T2D showed a similar pattern of concordance as for the earlier Finnish study, with $41 \%$ probandwise and $34 \%$ pairwise for $M Z$ and $19 \%$ probandwise and $12 \%$ pairwise for DZ twins. Over the follow-up period of up to 28 years, $\sim 50 \%$ of $\mathrm{MZ}$ co-twins subsequently became type 2 diabetic while the rate for $\mathrm{DZ}$ was approximately one third (Lehtovirta et al., 2010). At present, few studies have applied the discordant design to T2D. Using a longitudinal discordant design, Kujala et al. (2000) showed that lifestyle factors may influence a genetic pre-disposition for diabetes, as physically active twins developed diabetes less often than their sedentary co-twins. Ribel-Madsen et al. (2012) observed small quantitative differences in DNA methylation in skeletal muscle and adipose tissue in T2D discordant MZ twins, while Bork-Jensen et al. (2015) found differences in T2D discordant MZ pairs in the regulation of several microRNAs. Furthermore, acquired obesity has been shown to significantly contribute to insulin resistance and pre-diabetes in weight-discordant MZ twin pairs (Naukkarinen et al., 2012).

Here, we examine the T2D prevalence, concordance pattern, and heritability in same-sex twin pairs aged 45 years and older, in twin cohorts from seven European countries (Denmark, Finland, Norway, the Netherlands, Spain, Sweden, and the United Kingdom) and from Australia, and identify T2D discordant MZ twin pairs for future in-depth genotyping, omics, and phenotyping studies.

\section{Materials and Methods}

\section{Participants and Cohorts}

Table 1 provides an overview of the number of complete twin pairs available for each of the twin cohorts, as well as their average age. All cohorts that provided data for this paper have been described in detail elsewhere, but a brief description of each register is provided below.

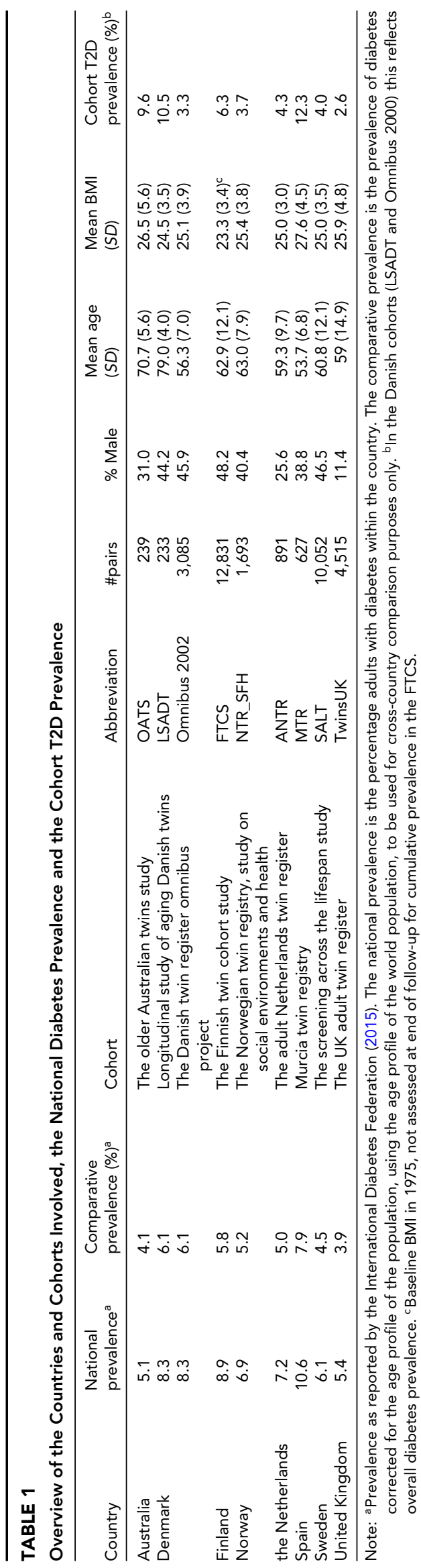


The Older Australian Twins Study (OATS) is a multicenter, longitudinal study that commenced in 2007. Study participants are identical and non-identical twin pairs aged 65 years and older living in the three eastern states of Australia who completed a comprehensive face-to-face assessment, including data on demographic, psychiatric, neuropsychological, and medical measures. The initial cohort comprised 623 individuals (including 284 twin pairs), and 450 participants completed a follow-up assessment at two years. For the majority of participants, DNA was extracted from peripheral blood samples and/or saliva samples. For more details, see Sachdev et al. (2013).

The Longitudinal Study of Aging Danish Twins (LSADT) is a longitudinal survey among Danish twins aged 70 years and older, followed every second year for up to six times from 1995-2005. In 1995, the twins were aged 75 years or older, but intake age was progressively decreased to age 70 years in the 1999 survey. A total of 4,731 twins have completed an intake LSADT assessment comprising 1,152 pairs. Approximately $80 \%$ of the participants have provided blood spots and 689 twins, comprising 290 same-sexed pairs, provided full blood samples in 1997 (Skytthe et al., 2013). The data in the present study were based on intact pairs who participated in survey 2005.

The Danish Twin Register Omnibus Project ('Omnibus 2002') is a longitudinal survey among the twin birth cohorts 1953-1982 from the population-based Danish Twin Registry. Participants received a nationwide questionnaire about physical health and health-related behavior in 1994 and a follow-up survey in 2002. As most of the participants reported no diabetes in the 1994 survey, the follow-up from 2002 was used to obtain the numbers of discordant pairs (Fejer et al., 2006).

The Finnish Twin Cohort Study (FTCS). The older cohort of the Finnish Twin Cohort Study consists of same-sex twin pairs born before 1958 and initially recruited in 1975. Follow-up questionnaires have been sent in 1981, 1990, and 2011 (Kaprio, 2013), and the twins have been linked to national medical registers (hospital discharge, medication, and cause-of-death data). Information from these registers, hospital charts, and questionnaire surveys were used to identify and classify twins with diabetes and the type of diabetes. Cases up to the end of 2004 were identified. For details, see Lehtovirta et al. (2010).

The Norwegian twin registry study on social factors and health (NTR_SFH). The NTR was established in 2009 as a merger of three major population-based Norwegian Twin Panels (Nilsen et al., 2012). It is housed at the Norwegian Institute of Public Health (NIPH) in Oslo. The NTR contains approximately 30,000 consenting twins, covering birth years 1896-1960 and 1967-1991, and has conducted studies in several areas including social factors, coronary heart disease, and its risk factors, mental health, dementia, cancer, and the epigenetics of autoimmune diseases (Nilsen et al., 2013). Among its strengths are linkage opportunities with nationwide health and socio-economic registries. The NTR data reported here are from a recent study conducted by Dr Harris on nearly 6,000 twins aged from $40-80$ years, to study social factors and health.

The Adult Netherlands Twin Register (ANTR) is an ongoing twin-family study on health-related behavior that assesses families with adolescent and (young) adult twins since 1991 and includes data on more than 22,000 individuals. Participants are invited every two or three years to complete a survey that contains questions about health, lifestyle, personality, and psychopathology. In addition, participants have been invited to provide DNA. For more details, see Willemsen et al. (2013) and http://www.tweelingenregister.org.

The Murcia Twin Registry (MTR) is a population-based register of adult twins in the region of Murcia, located in south-east Spain. To date, the MTR has identified 2,281 participants born between 1940 and 1966. Information is obtained longitudinally by face to face or telephone interviews and includes data on demographics, health, lifestyle, and related variables. In addition, biological samples (DNA) have been collected in a subset of the sample. For more details, see Ordoňana et al. (2013).

The Screening Across the Lifespan of Twins Study (SALT). Data were collected during a computer-assisted telephone interview between 1998-2002 on Swedish twins born 1958 or earlier. Introductory letters describing the study were sent to a random sample of approximately 1,000 twin pairs each month. A checklist of common illnesses, prescription, and non-prescription medication use was included in the study, and special emphasis was put on diagnostic items that could determine whether a twin was likely to have a disease (rather than simply asking the twin whether they have a disease). Items were presented in a branching format such that individuals were asked follow-up items (within item domain) if they responded positively to key introductory items. In total, 44,919 twin individuals participated in the SALT study (Lichtenstein et al., 2002; 2006).

The UK adult twin register (TwinsUK). The TwinsUK Resource is an active, nationwide adult twin registry based within the Department of Twin Research \& Genetic Epidemiology, at the St. Thomas' Hospital campus, King's College London. The resource recruits voluntary MZ and DZ same-sex twins throughout the country via media drives, and the registry currently has $\sim 13,000$ registered twins. These twins are predominantly healthy, female (83\%), with equal numbers of MZ (51\%) and DZ (49\%), and mostly middle-aged and older (range 18-103; mean 61 years). The database has an extensive range of phenotypes obtained via questionnaires, and over 7,000 have attended detailed 
clinical visits in the department over this period, enabling blood/urine/tissue sample collection, with many visiting at multiple time-points from 1992 onwards ( 700 with four visits). These resources are used to study the genetic and environmental etiology of complex diseases, as well as the healthy aging process (see Moayyeri, Hammond, Hart et al., 2013; Moayyeri, Hammond, Valdes et al., 2013; and http://www.twin-research.ac.uk).

\section{Type 2 Diabetes Phenotyping}

Data were included if participants were aged 45 years or over and did not report Type 1 diabetes. T2D was established in most cohorts (OATS, ANTR, SALT, NTR_SFH, TwinsUK) by questions in a survey asking whether a doctor ever diagnosed diabetes and whether any diabetes-related medication was used. In some cohorts, additional information was available from national/regional health registers (FTC, MTR, TwinsUK) or fasting blood glucose and/or insulin levels (OATS, ANTR). The exceptions were the Danish cohorts (LSADT, Omnibus 2002) where participants were asked to indicated whether they had diabetes with no distinction of type.

\section{Analyses}

Descriptive and genetic analyses were based on summary data on prevalence and twin concordance and discordance, provided by the individual cohorts. Meta-analysis of the concordance data was carried out using structural equation modeling in Mx (Neale et al., 2003). Data on the number of pairs concordant and discordant for T2D were the basis to estimate tetrachoric correlations for $\mathrm{MZ}$ and $\mathrm{DZ}$ twins, assuming an underlying normal liability distribution (Falconer \& Mackay, 1996), where the two categories are the result of a cut-off or threshold reflecting the prevalence of the trait (i.e., above the threshold T2D is indicated). Next, the contribution of additive genetic (A), common environmental (C), and unique environmental (E) influences to the variance in T2D liability was estimated by specifying a model in which A factors are correlated unity in $\mathrm{MZ}$ twin pairs and in DZ twins 0.50. Common environmental factors are correlated one in $\mathrm{MZ}$ and $\mathrm{DZ}$ twin pairs. The contribution of unique environment is obtained by constraining the sum of the squared path loadings due to A, C, and E to unity (Rijsdijk \& Sham, 2002). The number of estimated parameters within the model is three (one threshold and two path loadings). To test for heterogeneity, data from all cohorts were simultaneously analyzed. First, estimates for thresholds and path loadings were allowed to differ and next, path loadings were constrained to be equal across the cohorts. Within this model, 11 parameters (nine thresholds and two path loadings) were estimated. At a difference of 16 degrees of freedom (comparing the combined model with 11 parameters to the sum of the nine individual models with three parameters each), heterogeneity is implied if the difference in chi-square between the model and the total of the individual cohort models exceeds 26.296.

\section{Results}

Table 1 shows the distribution of the total number of twin pairs $(34,166$ pairs $)$ across the participating cohorts. In addition, the table provides the national prevalence and the comparative prevalence (the prevalence adjusted for the age profile of the nation) for each of the countries involved, as obtained from the International Diabetes Federation (IDF, 2015), and the cohort prevalence, as calculated based on the occurrence of diabetes in the same-sex twin pairs. The T2D prevalence data show a clear increase with age and BMI for T2D, but also reveal country differences, with Spain showing the highest and the United Kingdom showing the lowest rates of diabetes. The twin cohort prevalence broadly reflects the pattern that is seen in the standardized figures from the IDF, though some cohorts may have increased or decreased rates, possibly due to the age range of the participants or the varying criteria used for diagnosis.

Table 2 provides information on the number of $\mathrm{MZ}$ and DZ pairs and the rates of T2D concordance and discordances by country. Overall, the majority of the twin pairs (87.6-97.2\%) are concordant for not having T2D, and the concordance rates for having T2D are lower than for being T2D discordant. The T2D discordance rates differ across the cohorts, ranging from $2.9-11.2 \%$ for the MZ pairs and from $4.9-13.5 \%$ in same-sex DZ pairs, while discordance rates are always lower in $\mathrm{MZ}$ than in $\mathrm{DZ}$ twin pairs in all countries. Collectively, there are 716 (5.1\%) discordant MZ twin and 1,619 (8.0\%) discordant same-sex DZ twin pairs. Cohorts with more old age individuals and a higher T2D prevalence contribute relatively more to the number of T2D discordant pairs.

Collectively, there are $716(5.1 \%)$ discordant MZ twin and 1,619 (8.0\%) discordant same-sex DZ twin pairs. Cohorts with more old age individuals and a higher T2D prevalence contribute relatively more to the number of T2D discordant pairs.

Table 3 presents the results of genetic analyses. The MZ correlations are remarkably similar across the cohorts, with six of the eight correlations ranging within $0.72-0.78$, and while the DZ correlations show more variation, five of the eight are within the range of $0.36-0.47$. With respect to the ACE modeling, again a consistent pattern emerges. In all cohorts but LSADT, there is moderate to high heritability in the liability to T2D, with estimations for the larger cohorts ranging from $64 \%$ to $79 \%$. The influence of common environment is only suggested in the smaller cohorts, where the large confidence intervals indicate the uncertainty about these estimates. When analyzing all cohort data simultaneously and equating the path loadings from the ACE factors, the chi-square for the model was 31.31 . The chi-square 
TABLE 2

The Number and Percentage of Twin Pairs Concordant and Discordant for Having T2D Diabetes for Each of the Cohorts

\begin{tabular}{|c|c|c|c|c|c|c|c|c|c|c|c|c|c|c|}
\hline \multirow[b]{3}{*}{ Cohort } & & & \multicolumn{4}{|c|}{ Concordant no. T2D } & \multicolumn{4}{|c|}{ Concordant T2D } & \multicolumn{4}{|c|}{ Discordant T2D } \\
\hline & \multicolumn{2}{|c|}{ Number of pairs } & \multicolumn{2}{|c|}{$M Z$} & \multicolumn{2}{|c|}{$\mathrm{DZ}$} & \multicolumn{2}{|c|}{$\mathrm{MZ}$} & \multicolumn{2}{|c|}{$\mathrm{DZ}$} & \multicolumn{2}{|c|}{$\mathrm{MZ}$} & \multicolumn{2}{|c|}{$\mathrm{DZ}$} \\
\hline & $\mathrm{MZ}$ & DZ & $N$ & $\%$ & $N$ & $\%$ & $N$ & $\%$ & $N$ & $\%$ & $N$ & $\%$ & $N$ & $\%$ \\
\hline OATS & 159 & 80 & 135 & 84.9 & 68 & 85.0 & 8 & 5.0 & 2 & 2.5 & 16 & 10.1 & 10 & 12.5 \\
\hline LSADT & 107 & 126 & 92 & 86.0 & 102 & 81.0 & 3 & 2.8 & 7 & 5.6 & 12 & 11.2 & 17 & 13.5 \\
\hline Omnibus 2002 & 1,190 & 1,895 & 1,131 & 95.0 & 1,775 & 93.7 & 15 & 1.3 & 7 & 0.4 & 44 & 3.7 & 113 & 6.0 \\
\hline FTCS & 4,021 & 8,810 & 3,613 & 89.9 & 7,833 & 88.9 & 113 & 2.8 & 109 & 1.2 & 295 & 7.3 & 868 & 9.9 \\
\hline NTR_SFH & 854 & 839 & 797 & 93.3 & 791 & 94.3 & 16 & 1.9 & 4 & 0.5 & 41 & 4.8 & 44 & 5.2 \\
\hline ANTR & 644 & 247 & 596 & 92.5 & 231 & 93.5 & 13 & 2.0 & 0 & 0 & 35 & 5.4 & 16 & 6.5 \\
\hline MTR & 305 & 322 & 254 & 83.3 & 263 & 81.7 & 27 & 8.9 & 17 & 5.3 & 24 & 7.9 & 42 & 13.0 \\
\hline SALT & 4,212 & 5,840 & 3,963 & 94.1 & 5,389 & 92.3 & 72 & 1.7 & 42 & 0.7 & 177 & 4.2 & 409 & 7.0 \\
\hline TwinsUK & 2,478 & 2,037 & 2,382 & 96.1 & 1,930 & 94.7 & 24 & 1.0 & 7 & 0.3 & 72 & 2.9 & 100 & 4.9 \\
\hline Overall & 13,970 & 20,196 & 12,963 & 92.8 & 18,382 & 91.0 & 291 & 2.1 & 195 & 1.0 & 716 & 5.1 & 1,619 & 8.0 \\
\hline
\end{tabular}

TABLE 3

Twin Correlations, the Percentage Explained Variance by A, C, and E for the Full Model and the Difference in Fit Function Between the Individual Models and the Combined Model in Which the ACE Estimates Were Equated for the Cohorts

\begin{tabular}{|c|c|c|c|c|c|c|c|c|c|}
\hline \multirow[b]{2}{*}{ Cohort } & \multicolumn{2}{|c|}{ Number of pairs } & \multicolumn{2}{|c|}{ Twin correlations } & \multicolumn{3}{|c|}{ ACE } & \multicolumn{2}{|c|}{$\begin{array}{l}\text { Difference in } \\
\text { fit function }\end{array}$} \\
\hline & $\mathrm{MZ}$ & DZ & $\mathrm{MZ}$ & DZ & A & C & E & $\mathrm{MZ}$ & DZ \\
\hline OATS & 159 & 80 & $0.74(0.46-0.90)$ & $0.47(-0.10-0.83)$ & $0.51(0.00-0.90)$ & $0.22(0.00-0.82)$ & $0.27(0.10-0.55)$ & 0.05 & 0.09 \\
\hline LSADT & 107 & 126 & $0.55(0.08-0.85)$ & $0.69(0.37-0.88)$ & $0.00(0.00-0.69)$ & $0.64(0.06-0.82)$ & $0.36(0.14-0.61)$ & 1.05 & 3.12 \\
\hline $\begin{array}{c}\text { Omnibus } \\
2002\end{array}$ & 1,190 & 1,895 & $0.76(0.61-0.86)$ & $0.29(0.09-0.48)$ & $0.74(0.43-0.85)$ & $0.00(0.00-0.25)$ & $0.26(0.15-0.40)$ & -0.05 & 0.48 \\
\hline FTCS & 4,021 & 8,810 & $0.72(0.66-0.77)$ & $0.38(0.32-0.44)$ & $0.66(0.49-0.77)$ & $0.06(0.00-0.19)$ & $0.29(0.24-0.34)$ & 2.27 & 0.27 \\
\hline NTR_SFH & 854 & 839 & $0.76(0.61-0.87)$ & $0.41(0.12-0.65)$ & $0.64(0.08-0.86)$ & $0.11(0.00-0.61)$ & $0.25(0.14-0.40)$ & 0.04 & 0.03 \\
\hline ANTR & 644 & 247 & $0.74(0.57-0.86)$ & $0.15(-0.48-0.67)$ & $0.73(0.00-0.85)$ & $0.00(0.00-0.72)$ & $0.27(0.15-0.45)$ & 0.01 & 0.20 \\
\hline MTR & 305 & 322 & $0.89(0.78-0.95)$ & $0.65(0.45-0.79)$ & $0.47(0.11-0.89)$ & $0.42(0.01-0.72)$ & $0.11(0.05-0.22)$ & 5.53 & 5.87 \\
\hline SALT & 4,212 & 5,840 & $0.78(0.72-0.83)$ & $0.39(0.30-0.48)$ & $0.79(0.58-0.83)$ & $0.00(0.00-0.18)$ & $0.21(0.17-0.27)$ & 0.15 & 0.17 \\
\hline TwinsUK & 2,478 & 2,037 & $0.77(0.66-0.85)$ & $0.36(0.15-0.54)$ & $0.77(0.43-0.85)$ & $0.00(0.00-0.30)$ & $0.23(0.15-0.33)$ & 0.95 & 0.14 \\
\hline Combined & 13,970 & 20,196 & $0.76(0.73-0.79)$ & $0.41(0.37-0.45)$ & $0.72(0.61-0.78)$ & $0.04(0.00-0.13)$ & $0.25(0.22-0.28)$ & 9.99 & 10.37 \\
\hline
\end{tabular}

estimates for the individual models added up to 10.95 , resulting in a chi-square difference of 20.18 which, at a difference of 16 degrees of freedom, indicates there is no significant evidence for heterogeneity. To explore the difference in chi-square more closely, the difference in fit function between the individual models and the combined model was examined (see Table 3): more than $50 \%$ of the difference in chi-square is accounted for by the MTR. Overall, the results show that additive genetic effects account for the majority $(72 \%)$ of the variance in liability to T2D.

\section{Discussion}

Although the discordance for T2D within MZ twin pairs is relatively low, the DISCOTWIN consortium is able, by combining the resources of twin cohorts, to identify a substantial number of T2D discordant MZ pairs. The collaborative effort of DISCOTWIN also allows for good estimates of T2D heritability and $\mathrm{MZ}$ concordance, which are needed as the previous literature has been inconsistent. The data collected from different countries were surprisingly consistent, with all cohorts reporting over $87 \%$ concordance in $\mathrm{MZ}$ pairs, due mostly to both twins reporting no T2D. When one of the twins reported T2D, in $20-53 \%$ of the MZ pairs the cotwin also reported T2D, while this was much lower (0-29\%) for DZ twin pairs. The increasing prevalence of obesity as the main risk factor for T2D may be responsible for further increases in rates of concordance. In a way, the concordance rate is perhaps the upper limit for the potential of predictive strategies for personalized medicine, and high concordance rates for T2D are therefore encouraging for future efforts at early prediction using omics.

The numbers observed in the EURODISCOTWIN consortium will be sufficient to look at more complex interactions and follow-up on findings by replication and indepth analyses. The current overview is pre-dominantly based on survey data and thus reflects the starting point for twin pair selection. Most twin registers are able to contact their twin participants for additional phenotyping or can link to national health register or population databases to obtain more refined diagnoses. For selected twin pairs, T2D status can therefore be verified, for instance, by fasting glucose or glucose tolerance tests, or via doctor diagnosis in patient records. A thorough verification of the type of diagnoses may still be necessary, even when excluding Type 1 diabetes cases and restricting the sample to age 
45 years and over, as late-onset autoimmune diabetes of adulthood (sometimes called LADA, slow onset diabetes or diabetes type 1.5) may occur frequently and is often mistakenly taken for T2D (Landin-Olsson, 2002). It should be noted that the recruitment method and type of data available may also influence the discordance rates. For instance, register data may not cover all twins in the population, while those relying on hospital records will have an underrepresentation of cases. When comprehensive medication data are available (as in the Nordic countries), all medicated cases will be identified, but unless survey data are available, non-medicated individuals will be missed. Many non-medicated cases will become registered in the healthcare system and receive medication over time. Hence, models that take into account the lifecourse and development of T2D as well as censoring and competing causes of death will provide more sophisticated estimates of the overall contribution of genetic factors to the disease. Within the DISCOTWIN consortium, a wide range of such selection strategies across the countries are available, with sometimes the possibility of combining strategies. In addition to the possibility of follow-up on diagnoses and the acquisition of new genotypic and phenotypic data in identified pairs, twin registers generally have already followed their twin participants for a number of years, thereby allowing investigations into lifestyle and other behavioral differences in the T2D discordant pairs, using earlier (longitudinal) data. Establishing a format for information collection in discordant twin pairs, once they are identified, will result in an evergrowing informative database, as more twins reach the age at which T2D may be expected to develop, with data preceding and following T2D development. The follow-up of discordant T2D pairs may be particularly important, since the non-T2D twin is at increased risk of developing T2D, as may be evident from the higher T2D concordance in the older twin cohorts and as documented by Lehtovirta et al. (2010).

The contingency tables allowed for an estimation of the heritability that provided a consistent pattern across the cohorts. Heterogeneity was not implied, and using the combined data the heritability for T2D was estimated at $72 \%$. This is in line with the upper limit (69\%) reported by Almgren et al. (2011) when the age range was restricted to 35-60 years. In the present study, data were restricted to twin pairs who were at least 45 years of age at the time the T2D assessment was obtained, to ensure both twins had a chance to develop T2D. It is likely that age differences contribute to the differences in heritability estimates for T2D across studies. The high heritability seen here for our twins further reduces the chances of identifying T2D discordant MZ pairs, thereby providing additional support for the establishment of the DISCOTWIN consortium to obtain sufficient numbers for more in-depth study.

The combination of phenotypic and genotypic information, especially when collected longitudinally, in these T2D discordant pairs can lead to important new insights into epigenetic processes. With regards to epigenome-wide association studies (EWAS), a population-based case-control methodology encounters significant issues, particularly due to the inherent power reduction because of the influence of genetic heterogeneity on the epigenome (Mill \& Heijmans, 2013). This genetic confounding effect may be direct, in cis or trans adding huge complexity, especially if size effects are small. Leveraging the virtually identical genetic backgrounds of $\mathrm{MZ}$ twins in a disease-discordant model has thus immense advantages in the hunt for disease-associated non-genetic factors. Accounting for these confounding genetic effects and additionally many shared environmental and cohort effects provides significantly greater power than other study designs (Rakyan et al., 2011), and these combined numbers across the consortium will enable rigorous replication of small scale positive findings, as well as novel discoveries to be made. Many other omics technologies may be applied to disease discordant twins (metabolomics, transcriptomics, glycomics, metagenomics, proteomics), providing a complete picture of the processes involved in disease development and progression (Pallister et al., 2014).

In conclusion, through collaboration, the twin registers can generate the numbers and data to conduct in-depth analyses of the (epi)genetic and environmental pathways that lead to twin differences in disease. With the discordant twin design as a powerful base, the DISCOTWIN consortium can provide new insights that may lead to prognostic, predictive, and therapeutic targets for the prevention and treatment of a wide range of diseases, such as T2D.

\section{Acknowledgments}

OATS would like to acknowledge access to the Australian Twin Registry, a national resource supported by an Enabling Grant (ID 628911) from the Australian National Health \& Medical Research Council (NHMRC). OATS is supported by a NHMRC/ARC Strategic Award Grant (ID 401162), and a NHMRC Project Grant (ID 1045325) and Program Grant (ID 350833). Karen Mather is supported by an Alzheimer's Australia Dementia Research Foundation Postdoctoral Fellowship. We gratefully thank all our participants, and wish to acknowledge the OATS research team and Chief Investigators, in particular Julian Trollor, Henry Brodaty, David Ames, Nick Martin, Margie Wright, Katherine Samaras, Teresa Lee, and Wei Wen. LSADT was supported by the National Institute on Aging grant P01 AG08761. The Danish Omnibus survey was supported by The Danish Medical Research Council and the Novo Nordic Foundation. The FTCS is funded by Academy of Finland grants, 265240, 263278 to JK and 272376 and 266286 to KP, and Novo Nordisk Foundation (KP). Data from the Norwegian Twin Registry is supported, in part, from the European Union's Seventh Framework Programs ENGAGE 
Consortium (grant agreement HEALTH-F4-2007-201413, and BioSHaRE EU (grant agreement HEALTH-F4-2010261433). ANTR was supported by the Netherlands Organization for Scientific Research (900-562-137, 904-61090, 985-10-002, 904-61-193, 56-464-14192, 400-03-330, 480-04-004, 400-07-080, 911-09-032, 451-06-004, 451-08026, 451-10-005), the Netherlands Organization for Health Research and Development (ZonMW 3100.0038, 940-37024, 31160008), EMGO+ Institute for Health and Care Research, Neuroscience Campus Amsterdam, Center for Medical Systems Biology, BBMRI - NL (184.021.007: Biobanking and Biomolecular Resources Research Infrastructure), National Institutes of Health (NIH 5R37DA018673-03, R01 MH059160, 1RC2 MH089951- 01, 4R37DA01867306, 1R01 MH087646-01A1), National Institute of Mental Health (RFA MH08120), FP7 ENGAGE (FP7-HEALTHF42007-201413), European Research Council (230374-GMI, 284167). The MTR is funded by Fundación Séneca, Murcia, Spain (15302/PHCS/10) and grateful to the Murcia Health Council for data validation accessibility (CMBD and OMI-AP). SALT was supported by grants from the NIH (AG08724), The Swedish Council for Working Life and Social Research, and the Swedish Research Council. TwinsUK is funded by the Wellcome Trust and European Community's Seventh Framework Program (FP7/2007-2013). They also receive support from the National Institute for Health Research (NIHR)-funded BioResource, Clinical Research Facility and Biomedical Research Centre based at Guy's and St Thomas' NHS Foundation Trust in partnership with King's College London. Tim Spector is holder of an ERC Advanced Principal Investigator award. SNP Genotyping was performed by The Wellcome Trust Sanger Institute and National Eye Institute via NIH/CIDR.

\section{References}

Almgren, P., Lehtovirta, M., Isomaa, B., Sarelin, L., Taskinen, M. R., Lyssenko, V., ... Botnia Study Group. (2011). Heritability and familiality of type 2 diabetes and related quantitative traits in the Botnia Study. Diabetologia, 54, 28112819.

Barnett, A. H., Spiliopoulos, A. J., Pyke, D. A., Stubbs, W. A., Burrin, J., \& Alberti, K. G. (1981). Metabolic studies in unaffected co-twins of non-insulin-dependent diabetics. British Medical Journal, 282, 1656-1658.

Bonnefond, A., \& Froguel, P. (2015). Rare and common genetic events in type 2 diabetes: What should biologists know? Cell Metabolism, 21, 357-368.

Bork-Jensen, J., Scheele, C., Christophersen, D. V., Nilsson, E., Friedrichsen, M., Fernandez-Twinn, D. S., ... Vaag, A. (2015). Glucose tolerance is associated with differential expression of microRNAs in skeletal muscle: results from studies of twins with and without type 2 diabetes Diabetologia, 58, 363-373.

Buchwald, D., Kaprio, J., Hopper, J. L., Sung, J., Goldberg, J., Fortier, I., ... Harris, J. R. (2014). International network of twin registries (INTR): Building a platform for international collaboration. Twin Research and Human Genetics, $17,574-577$.

Committee on Diabetic Twins. (1988). Japan diabetes society diabetes mellitus in twins: A cooperative study in Japan. Diabetes Research and Clinical Practice, 5, 271-280.

De Moor, M. H., Boomsma, D. I., Stubbe, J. H., Willemsen, G., \& de Geus, E. J. (2008). Testing causality in the association between regular exercise and symptoms of anxiety and depression. Archives of General Psychiatry, 65, 897-905.

European Commission. (2004). Quality of Life and Management of the Living Resource, Fifth Framework Program 19982002. Luxembough: Author.

Falconer, D. S., \& Mackay, T. F. C. (1996). Introduction to quantitative genetics. Essex, UK: Pearson Educational Limited.

Fejer, R., Hartvigsen, J., \& Kyvik, K. O. (2006). Heritability of neck pain: A population-based study of 33,794 Danish twins. Rheumatology, 45, 589-594.

Friedman, J. M. (2009). Obesity: Causes and control of excess body fat. Nature, 459, 340-342.

Gluckman, P. D., Hanson, M., Zimmet, P., \& Forrester, T. (2011). Losing the war against obesity: The need for a developmental perspective. Science Translational Medicine, 27, $93 \mathrm{~cm} 19$.

Hur, Y. M., \& Craig, J. M. (2013). Twin registries worldwide: An important resource for scientific research. Twin Research and Human Genetics, 16, 1-12.

International Diabetes Federation (IDF). (2015). Diabetes atlas (6th ed.). Retrieved March 20, 2015, from http://www.idf.org/atlasmap/atlasmap.

Kaprio, J. (2013). The finnish twin cohort study: An update. Twin Research and Human Genetics, 16, 157-162.

Kaprio, J., Tuomilehto, J., Koskenvuo, M., Romanov, K., Reunanen, A., Eriksson, J., ... Kesäniemi, Y. A. (1992). Concordance for type 1 (insulin-dependent) and type 2 (non-insulin-dependent) diabetes mellitus in a populationbased cohort of twins in Finland. Diabetologia, 35, 10601067.

Kettunen, J., Perola, M., Martin, N. G., Cornes, B. K., Wilson, S. G., Montgomery, G. W., ... GenomEUtwin-project. (2009). Multicenter dizygotic twin cohort study confirms two linkage susceptibility loci for body mass index at 3q29 and $7 \mathrm{q} 36$ and identifies three further potential novel loci. International Journal of Obesity, 33, 1235-1242.

Korja, M., Silventoinen, K., McCarron, P., Zdravkovic, S., Skytthe, A., Haapanen, A., ... GenomEUtwin Project. (2010). Genetic epidemiology of spontaneous subarachnoid hemorrhage: Nordic Twin Study. Stroke, 41, 24582462.

Kujala, U. M., Kaprio, J., \& Koskenvuo, M. (2000). Diabetes in a population-based series of twin pairs discordant for leisure sedentariness. Diabetologia, 43, 259.

Kujala, U. M., Kaprio, J., \& Koskenvuo, M. (2002). Modifiable risk factors as predictors of all-cause mortality: The roles of genetics and childhood environment. American Journal of Epidemiology, 156, 985-993. 
Landin-Olsson, M. (2002). Latent autoimmune diabetes in adults. Annals of the New York Academy of Sciences, 958, 112-116.

Lehtovirta, M., Pietiläinen, K. H., Levälahti, E., Heikkilä, K., Groop, L., Silventoinen, K., ... Koskenvuo, M. (2010). Evidence that BMI and type 2 diabetes share only a minor fraction of genetic variance: A follow-up study of 23,585 monozygotic and dizygotic twins from the Finnish Twin Cohort Study. Diabetologia, 53, 1314-1321.

Lichtenstein, P., deFaire, U., Floderus, B., Svartengren, M., Svedberg, P., \& Pedersen, N. L. (2002). The Swedish twin registry: A unique resource for clinical, epidemiological and genetic studies. Journal of Internal Medicine, 252, 184205.

Lichtenstein, P., Sullivan, P. F., Cnattingius, S., Gatz, M., Johansson, S., Carlström, E., ... Pedersen, N. L. (2006). The Swedish twin registry in the third millennium: An update. Twin Research and Human Genetics, 9, 875982.

McCarthy, M. I. (2010). Genomics, type 2 diabetes, and obesity. New England Journal of Medicine, 363, 23392350 .

Medici, F., Hawa, M., Ianari, A., Pyke, D. A., \& Leslie, R. D. (1999). Concordance rate for type II diabetes mellitus in monozygotic twins: Actuarial analysis. Diabetologia, 42, 146-150.

Mill, J., \& Heijmans, B. T. (2013). From promises to practical strategies in epigenetic epidemiology. Nature Reviews Genetics, 14, 585-594.

Moayyeri, A., Hammond, C. J., Hart, D. J., \& Spector, T. D. (2013). The UK adult twin registry (TwinsUK Resource). Twin Research and Human Genetics, 16, 144-149.

Moayyeri, A., Hammond, C. J., Valdes, A. M., \& Spector, T. D. (2013). Cohort profile: TwinsUK and healthy ageing twin study. International Journal of Epidemiology, 42, 76-85.

Mulder, E. J., Van Baal, C., Gaist, D., Kallela, M., Kaprio, J., Svensson, D. A., ... Palotie, A. (2003). Genetic and environmental influences on migraine: A twin study across six countries. Twin Research, 6, 422-431.

Naukkarinen, J., Rissanen, A., Kaprio, J., \& Pietiläinen, K. H. (2012). Causes and consequences of obesity: The contribution of recent twin studies. International Journal of Obesity, 36, 1017-1024.

Neale, M. C., Boker, S. M., Xie, G., \& Maes, H. H. (2003). Mx: Statistical modelling (6th ed.), Richmond, VA: Department of Psychiatry, Virginia Commonwealth University.

Newman, B., Selby, J. V., King, M. C., Slemenda, C., Fabsitz, R., \& Friedman, G. D. (1987). Concordance for type 2 (noninsulin-dependent) diabetes mellitus in male twins. Diabetologia, 30, 763-768.

Nilsen, T. S., Brandt, I., Magnus, P., \& Harris, J. R. (2012). The Norwegian twin registry. Twin Research and Human Genetics, 15, 775-780.

Nilsen, T. S., Knudsen, G. P., Gervin, K., Brandt, I., Røysamb, E., Tambs, K., .. Harris, J. R. (2013). The Norwegian twin registry from a public health perspective: A research update. Twin Research and Human Genetics, 16, 285-295.
Ordoñana, J. R., Rebollo-Mesa, I., Carrillo, E., ColodroConde, L., García-Palomo, F. J., González-Javier, F., ... Pérez-Riquelme, F. (2013). The Murcia twin registry: A population-based registry of adult multiples in Spain. Twin Research and Human Genetics, 16, 302-306.

Pallister, T., Spector, T. D., \& Menni, C. (2014). Twin studies Advance the understanding of gene-environment interplay in human nutrigenomics. Nutrition Research Reviews, 27, 242-251.

Peltonen, L., \& GenomEUtwin, . (2003). GenomEUtwin: A strategy to identify genetic influences on health and disease. Twin Research, 6, 354-360.

Perola, M., Sammalisto, S., Hiekkalinna, T., Martin, N. G., Visscher, P. M., Montgomery, G. W., ... GenomEUtwin Project. (2007). Combined genome scans for body stature in 6,602 European twins: Evidence for common Caucasian loci. PLoS Genetics, 3, e97.

Poulsen, P., Kyvik, K. O., Vaag, A., \& Beck-Nielsen, H. (1999). Heritability of type II (non-insulin-dependent) diabetes mellitus and abnormal glucose tolerance - A populationbased twin study. Diabetologia, 42, 139-145.

Rakyan, V. K., Down, T. A., Balding, D. J., \& Beck, S. (2011). Epigenome-wide association studies for common human diseases. Nature Reviews Genetics, 12, 529-541.

Ribel-Madsen, R., Fraga, M. F., Jacobsen, S., Bork-Jensen, J., Lara, E., Calvanese, V., ... Poulsen, P. (2012). Genomewide analysis of DNA methylation differences in muscle and fat from monozygotic twins discordant for type 2 diabetes. PLoS One, 7, e51302.

Rijsdijk, F., \& Sham, P. (2002). Analytic approaches to twin data using structural equation models. Briefings in Bioinformatics, 3, 119-133.

Sachdev, P. S., Lee, T., Wen, W., Ames, D., Batouli, A. H., Bowden, J., ... OATS Research Team. (2013). The contribution of twins to the study of cognitive ageing and dementia: The older Australian twins study. International Review of Psychiatry, 25, 738-747.

Schousboe, K., Willemsen, G., Kyvik, K. O., Mortensen, J., Boomsma, D. I., Cornes, B. K., ... Harris, J. R. (2003). Sex differences in heritability of BMI: A comparative study of results from twin studies in eight countries. Twin Research, 6, 409-421.

Silventoinen, K., Sammalisto, S., Perola, M., Boomsma, D. I., Cornes, B. K., Davis, C., .. Kaprio, J. (2003). Heritability of adult body height: A comparative study of twin cohorts in eight countries. Twin Research, 6, 399-408.

Skytthe, A., Christiansen, L., Kyvik, K. O., Bødker, F. L., Hvidberg, L., Petersen, I., ... Christensen, K. (2013). The danish twin registry: Linking surveys, national registers, and biological information. Twin Research and Human Genetics, 16, 104-111.

Smith, C. (1970). Heritability of liability and concordance in monozygous twins. Annals of Human Genetics, 34, 85-91.

Stubbe, J. H., Boomsma, D. I., Vink, J. M., Cornes, B. K., Martin, N. G., Skytthe, A., ... de Geus, E. J. (2006). Genetic influences on exercise participation in 37,051 twin pairs from seven countries. PLoS One, 20, e22. 
Surakka, I., Whitfield, J. B., Perola, M., Visscher, P. M., Montgomery, G. W., Falchi, M., ... GenomEUtwin Project. (2012). A genome-wide association study of monozygotic twin-pairs suggests a locus related to variability of serum high-density lipoprotein cholesterol. Twin Research and Human Genetics, 15, 691-699.

van Dongen, J., Slagboom, P. E., Draisma, H. H., Martin, N. G., \& Boomsma, D. I. (2012). The continuing value of twin studies in the omics era. Nature Reviews Genetics, 13, 640653.

Vink, J. M., Boomsma, D. I., Medland, S. E., de Moor, M. H., Stubbe, J. H., Cornes, B. K., ... de Geus, E. J. (2011). Variance components models for physical activity with age as modifier: A comparative twin study in seven countries. Twin Research and Human Genetics, 14, 25-34.

Willemsen, G., Vink, J. M., Abdellaoui, A., den Braber, A., van Beek, J. H., Draisma, H. H., ... Boomsma, D. I. (2013). The adult Netherlands twin register: Twenty-five years of survey and biological data collection. Twin Research and Human Genetics, 16, 271-281.

World Health Organization (WHO). (2015). Diabetes Fact sheet No 312. Retrieved March 20, 2015, from http:// www.who.int/mediacentre/factsheets/fs312/en/.

Zimmet, P., Alberti, K. G., \& Shaw, J. (2001). Global and societal implications of the diabetes epidemic. Nature, 13, 782-787. 